Malaya Journal of Matematik, Vol. S, No. 1, 99-101, 2019

https://doi.org/10.26637/MJM0S01/0025

\title{
Partial differentiability on graphs
}

\author{
M. Regees ${ }^{1}$ and T. Sajitha Kumari ${ }^{2 *}$
}

\begin{abstract}
Operations play a vital role in the field of Mathematics. There are many operations by which new graphs are obtained from the old ones. In this paper we try to form a new graph from the old one through a new operation, partial differentiability. Let $G$ be a graph of order $n$. Consider an arbitrary vertex $v$ in $G$. We remove all edges of $G$ with respect to the vertex $v$. Now we consider another vertex $u$ in $G_{v}^{(1)}$ and remove all edges which are adjacent to $u$. The resultant graph is denoted by $G_{u}^{(2)}$. This is the partial derivative of $G_{v}^{(1)}$ with respect to $u$. The minimum of $r$ such that $G^{(r)} \cong m K_{1}$, is called the order of partial differentiation, denoted by $r(G)$, where $m$ is a positive integer. In this paper we introduced the partial differentiability of graphs.

\section{Keywords}

Graph, Differentiation, Partial differentiation.

\section{AMS Subject Classification}

$05 C 78$.

${ }^{1}$ Department of Mathematics, Malankara Catholic College, Kaliakavilai-629153, Tamil Nadu, India.

${ }^{2}$ Research Scholar, Department of Mathematics, Scott Christian College, Nagercoil- 629001, Tamil Nadu, India.

*Corresponding author: ${ }^{1}$ regeesregees@gmail.com; ${ }^{2}$ sajithasabu09@gmail.com

Article History: Received 21 December 2018; Accepted 11 February 2019
\end{abstract} which are adjacent to the vertex $v$. The resultant graph is denoted by $G_{v}^{(1)}$. This is called the partial differentiation

(C)2019 MJM.

\section{Contents}

1 Introduction .99

2

References

\section{Introduction}

Partial derivatives can be used to describe a wide variety of phenomena such as sound, heat, electrostatics, electrodynamics, fluid dynamics, elasticity, quantum mechanics etc. There has been a considerable effort to study differentiation on graphs. In [1] introduced the differentiability of graphs. This motivated to study partial differentiation and its properties on graphs.

\section{Main Results}

Definition 2.1. Let $G$ be a graph of order $n$. Consider an arbitrary vertex $v$ in $G$. Remove all edges which are adjacent to the vertex $v$. The resultant graph is denoted by $G_{v}^{(1)}$. This is called the partial derivative of $G$ with respect to $v$. Now consider another vertex $u$ in $G_{v}^{(1)}$ and remove all edges which are adjacent to $u$. The resultant graph is denoted by $G_{v}^{(2)}$. This is called the partial derivative of $G_{v}^{(1)}$ with respect to $u$.

The minimum of $r$ such that $G^{(r)} \cong m K_{1}$, is called the order of the partial differentiation; where $m$ is a positive integer and it is denoted by $r(G)$.

Remark 2.2. I. If $G$ is an empty graph, then $r(G)=0$.

2. All graphs are partial differentiable.

Example 2.3.

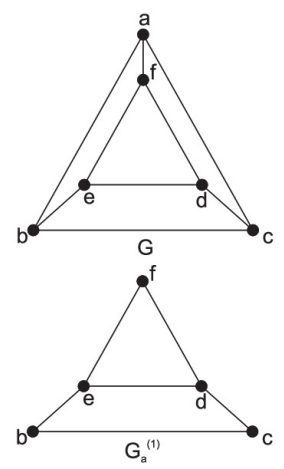




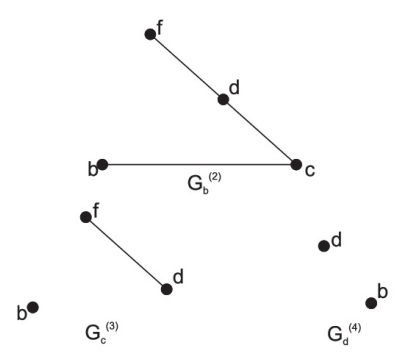

Definition 2.4. The adjacency matrix of a graph $G$ with $n$ vertices and no parallel edges is an $n$ by $n$ symmetric binary matrix $X=\left[x_{i j}\right]$, defined over the ring of integers such that

$$
x_{i j}=\left\{\begin{array}{l}
1, \text { if there is an edge between } i^{\text {th }} \text { and } j^{\text {th }} \text { vertices } \\
0, \quad \text { if there is no edges between them. }
\end{array}\right.
$$

\section{Finding order of partial differentiation by adjacency ma-} trix

\section{Algorithm:}

1. Start

2. Read an adjacency matrix, A and initialize count $=0$

3. Find the row / column which has maximum number of entries 1.

4. If the maximum entries 1 in two or more column / row are same choose any one arbitrarily.

5. Remove that column and row

6. Set count $=$ count +1

7. Repeat steps 2, 3,4 and 5 until we get matrix, A with all entries zero.

8. Order of partial differentiation is count.

9. Stop.

\section{Example 2.5.}

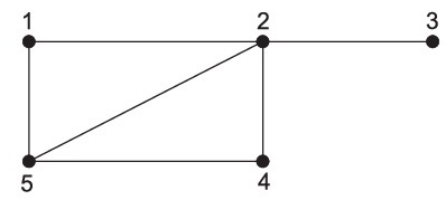

Adjacency matrix, $\left[a_{i j}\right]=$

$\begin{array}{lllll}1 & 2 & 3 & 4 & 5\end{array}$

$1\left(\begin{array}{lllll}0 & 1 & 0 & 0 & 1\end{array}\right)$

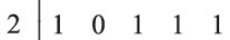

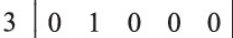

$4 \quad 0 \begin{array}{lllll}4 & 0 & 0 & 1\end{array}$

$5\left(\begin{array}{lllll}1 & 1 & 0 & 1 & 0\end{array}\right)$
Step 1

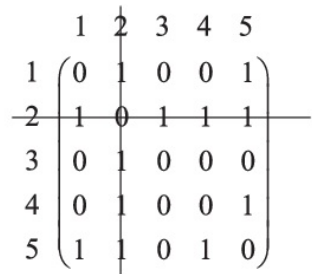

Step 2

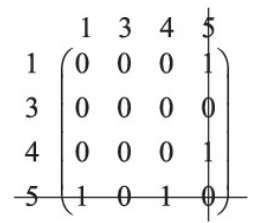

Here $r(G)=2$

Theorem 2.6. For a complete graph, $K_{n} ; n \geq, G^{(n-1)} \cong K_{1}$.

Proof. We prove this theorem by mathematical induction.

First we prove the theorem is true for $n=2$.

If $n=2$, there are two vertices say $v_{1}$ and $v_{2}$.

Differentiate $G$ partially with respect to $v_{1}$ (or $v_{2}$ ). Then it remains $v_{2}$ (or $v_{1}$ ) only.

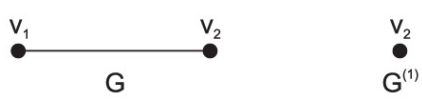

The theorem is true for $n=2$.

By assumption, that the theorem is true for $n=m$.

Hence, for $K_{m}, G^{(m-1)} \cong K_{1}$

Now we prove the theorem is true for $n=m+1$

Here $G$ contains $m+1$ vertices.

Let $v_{1}, v_{2}, v_{3}, \ldots, v_{m+1}$ be the vertices.

Differentiate $G$ partially with respect to an arbitrary vertex $v_{i}$.

The resultant graph is $K_{m}$.

We know that, for $K_{m}, G^{(m-1)} \cong K_{1}$

Hence for $K_{m+1}, G^{(m-1+1)} \cong K_{1}$

i.e., $G^{(m)} \cong K_{1}$

i.e., $G^{(n-1)} \cong K_{1} \quad(\because n=m+1)$.

Proposition 2.7. For a connected graph $G$ with $n$ vertices, $1 \leq r(G) \leq n-1$.

Proof. Let $\mathrm{G}$ be a connected graph with $\mathrm{n}$ vertices $v_{1}, v_{2}, v_{3}, \ldots, v_{n}$.

Let $v_{i}$ be an arbitrary vertex which is adjacent all other vertices and there exist no other edges between the remaining vertices. If we differentiate $G$ partially with respect to $v_{i}$, we get $r(G)=$ 1. Again let each vertex of $G$ be adjacent to all other vertices in $G$. Then by theorem $r(G)=n-1$.

Hence we get $1 \leq r(G) \leq n-1$. 


\section{References}

[1] G. Suresh Singh and V.N. Manju, Some Results on Differentiable Graphs, International Journal of Mathematical Archive, 9(4)(2019), 37-45.

[2] F. Harary, Graph Theory, Narosa Publishing House, Delhi, 1993.

[3] D. Narsingh, Graph Theory with Applications to Engineering and Computer Science, Prentice Hall of India Private Limited, 1979.

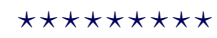

ISSN(P):2319 - 3786

Malaya Journal of Matematik

$\operatorname{ISSN}(\mathrm{O}): 2321-5666$

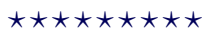

